\title{
Pattern Recognition of Process Mean Shift using Combined ANN Recognizer
}

\author{
Olatunde A. Adeoti \\ Department of Mathematics and Statistics \\ Bowen University, Iwo \\ Osun state, Nigeria
}

\author{
Rotimi F. Afolabi \\ Department of Mathematics and Statistics \\ Bowen University, Iwo \\ Osun state, Nigeria
}

\begin{abstract}
Artificial Neural Network (ANN) based model has been proposed for diagnosis of process mean shift. These are mainly generalized-based where only a single classifier was applied in the diagnosis of abnormal pattern. In this paper, we analyze the performance of a combined recognizer consisting of small-sized artificial neural networks on varying number of nodes in the hidden layer trained with Levenberg Marquardt and Quasi-Newton Algorithm. The results of our study illustrate the effectiveness of the combined recognizer and showed that combined recognizer performed better when number of hidden nodes is small, say, less than 15 in terms of recognition accuracies and mean square error as compared to the single recognizer.
\end{abstract}

Key words: : Bivariate Statistical Process Control; Combined ANN Recognizer; Pattern Recognition; Recognition Accuracy; Mean Square Error

\section{INTRODUCTION}

In many quality control environments, the process seldom involves several quality characteristics which are usually correlated and require an appropriate technique to monitor these characteristics. The use of multiple univariate control charts would be ineffective for controlling the process as it ignores the correlation between the variables and thus required the use of multivariate statistical methods. Several multivariate control charts have been developed to monitor and control multivariate process. These include the multivariate Shewhart chart based on the Hotelling's $\mathrm{T}^{2}$ statistic [8], MEWMA charts [10, 19], MCUSUM charts [4, 7 and 22] and Minimax control charts [20].

Monitoring and detecting mean shifts of multivariate process, though, can be achieved using multivariate control charts, the complexity of the control charts and the cross-correlation among variables make it difficult for analysis of out-ofcontrol signal as they always have difficulties in determining which variable or set of variables is (are) responsible for the signal when the process is out of control.

Different approaches have been developed by many researchers towards the detection and identification of abnormal process patterns. [1] proposed the univariate control chart with Bonferroni limits. [15] developed a method based on discriminant analysis to identify the out-of-control variables. He divided the complete set of variables into two subsets to determine which of the subsets is responsible for the out-of-control signal, but discriminating and knowing the subset suspected to be directly related to the cause of out-ofcontrol is subjective and depends on the practitioner. $[13,14]$ proposed the decomposition of $\mathrm{T}^{2}$ statistic and showed that the interpretation of a signal from a $\mathrm{T}^{2}$ statistic is greatly aided if the corresponding value is partitioned into independent parts. [5] used the multivariate control chart to detect the aberrant variables. The main idea of this method is the use of the univariate $t$ ranking procedure and it is based on $p$ unconditional $\mathrm{T}^{2}$ terms. [9] used the $\mathrm{T}^{2}$ control chart and principal components to detect the multivariate process by reducing the dimensionality of the process variables. But the identification of out-of-control variable with this method demands further knowledge of the process itself as the principal components do not always lead themselves to physical interpretation. [12] proposed a new method based on principal component analysis. These approaches to identify the abnormal patterns have several drawbacks as some of them are not easily interpretable in many cases and some contain extensive computation and sensitive to the number of variables.

Advances in computing technology have motivated many researchers to explore the use of artificial intelligence tools in statistical process control. One of such artificial intelligence tool is the Artificial Neural Network (ANN). Artificial Neural Network consists of a set of computational units called cells, neurons or nodes and a set of weighted, directed connections between these units. It is an adaptive, most often nonlinear system that learns to perform a function from data and has the ability to learn, recall and generalize knowledge. It is regarded as an important and emerging technique in pattern recognition of process patterns. [16] applied ANN recognizer for fault diagnosis of multivariate statistical process control where $\mathrm{T}^{2}$ charts and ANN model was utilized for monitoring process mean shift and identifying the variable(s) that is responsible for the mean shifts.

It must be stated that limited research work was reported on the combined recognizer approach for pattern recognition as most of the existing ANN model for pattern recognition are mainly generalized-based where only a single classifier was applied in the diagnosis of abnormal pattern. However, it is recognized that in general, the use of combined classifiers instead of a single classifier leads to an increase in the overall recognition accuracy $[17,21]$.Therefore further research on 
the combined recognizer towards pattern recognition is encouraged.

In this paper, we analyze the performance of a combined recognizer consisting of small-sized artificial neural networks on varying number of nodes in the hidden layer trained with Levenberg- Marquardt and Quasi-Newton Algorithm. The number of nodes was chosen empirically between 10 and 20 since there is no exact rule for chosen the number of nodes.

The paper is organized as follows: Section 2 presents the combined ANN recognition scheme. Section 3 describes the patterns and method of dataset generation. Section 4 to section 5 discusses combined ANN design, training, validation and testing. Section 6 is the results of study and discussions. Section 7 gives the conclusion

\section{COMBINED ANN RECOGNITION SCHEME}

A conceptual diagram of intended pattern recognition scheme is shown in figure 1 which consists of two phases. In Phase I, the multivariate statistical process control chart, precisely the Hotelling's $T^{2}$ statistic was utilized to perform process monitoring i.e. to detect an out-of-control signal. When the chart gives an out-of-control signal, the combined neural network was applied for performance diagnosis of the ANN recognizer in phase II. Raw data-based are employed in the ANN pattern recognition

\section{MODELLING OF BIVARIATE PROCESS PATTERNS}

Bivariate process is the simplest case of multivariate statistical process control where only two process variables are monitored dependently. In a statistically in-control state, samples for both process variables are i.i.d with mean zero and standard deviation one. However, as employed in this work, let $X_{1}=\left(x_{11}, x_{12}, \ldots . x_{1 m}\right)^{\prime}$ and $X_{2}=\left(x_{21}, x_{22, \ldots x} x_{2 m}\right)^{y}$ represent data streams for process variable 1 and variable 2 based on observation window of 450 samples (150 for each pattern) generated from the process patterns. Three bivariate process upward mean shifts with a moderate positive correlation are investigated. These are $(1,0)$ - shift in first variable while second variable remain stable; $(0,1)$ - First variable remain stable while second variable shifted and $(1,1)$ - shift in first and second variable.

\subsection{Data Generator}

The type of dataset available for training, validation and testing of ANN in the detection and identification of process patterns has a strong influence on the performance of the pattern recognizer. Only when the model is trained through suitable and appropriate dataset can it acquire the ability to recognize the patterns of process mean vector. Ideally, the dataset ought to be obtained from real life manufacturing process environment. Since they are not economically available, there is need for the generation of process data through simulation. The common approach adopted by previous researchers to generate shift dataset is based on predefined model of $[3,6]$. However, we simulate our dataset from bivariate normal distribution when the variables are correlated.

The simulation was implemented using MATLAB software. The in-control mean is assumed to be a zero vector. The variance-covariance matrix is assumed to be scaled so as to have unit variance for all components. We restrict our work to the shift in process mean vector only. Let $X \sim \mathbb{N}_{2}(\mu, \Sigma)$ denotes the bivariate normal distribution with mean $\mu$ and variance $\Sigma=\left[\begin{array}{ll}1 & \rho \\ \rho & 1\end{array}\right]$ where $\sigma_{i, j}=1$, for all $\mathrm{i}$ and $\sigma_{i j}=\rho$ for all $i \neq j$ where $\rho$ is the correlation value between the two variables in each pattern. The in-control mean is assumed to be a zero vector. A moderate correlation coefficient of 0.5 is used and the mean vector is shifted from zero to 1.5

\section{DESIGN OF COMBINED ANN RECOGNIZER}

This section describes the architecture of the proposed combined ANN scheme. The proposed method is similar to the recognition design of [16] but the generalized-based ANN was replaced by the multistructure neural network design proposed by [21] which has been shown to improve the accuracy of recognition. A multistructure neural network consists of a number of interconnected single structure neural networks which combine the strength of different recognition techniques of individual recognizer for monitoring and diagnosis. The number of nodes in the hidden layer of each neural network is selected empirically by varying the number of nodes between 10 and 20 for each of the training algorithm. In our design, two single structures each slightly different from one another (in terms of activation function and training algorithms) is employed so that they yield slightly different outputs to a given set of inputs (same inputs). Each individual neural network is first trained on the training dataset using two different training algorithms and activation function. A combining module (structure) then combine the output of the individual unit structure and select the best output as final result. Combining module (structure) could use averaging, voting (i.e. Plurality Voting, Majority Voting, Winners-TakeAll) and Bayesian method. However, in this study the averaging method due to [11] is used because it is simple, easier and efficient in terms of recognition accuracy 


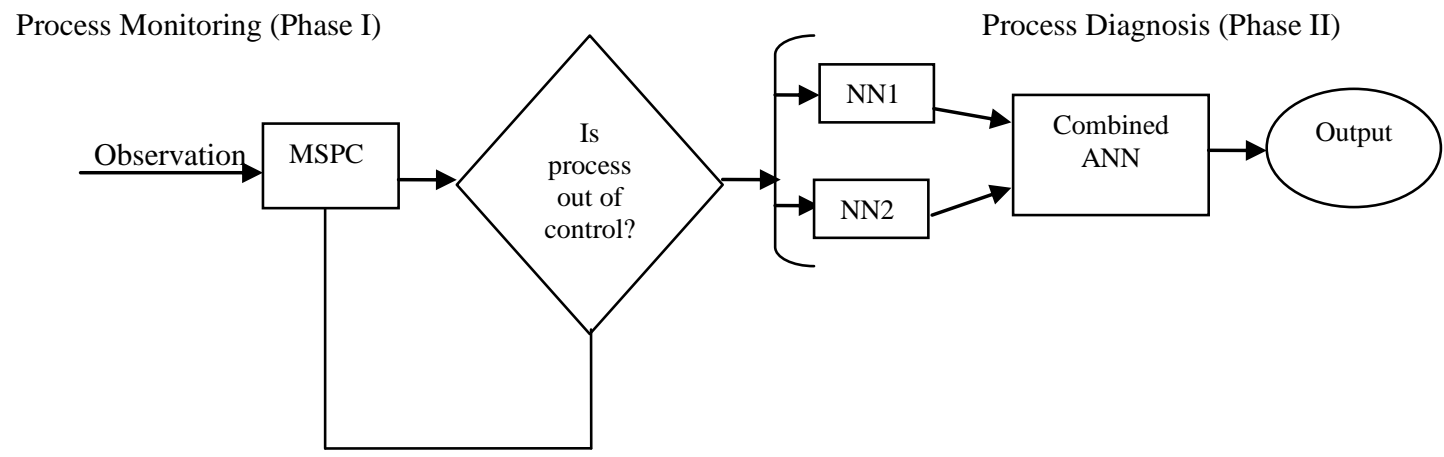

Fig 1: A conceptual diagram of MSPC-Combined ANN model

\section{ANN TRAINING, VALIDATION AND TESTING}

MATLAB 7.10 Neural network toolbox software was used for the training of the network. The network uses an epoch number of 5000. The number of nodes in the hidden layer of ANN architecture for the training algorithms is varied between 10 and 20. The performance error goal was set at 1E-008. Maximum number of validation failure was 25 . The learning rate was 0.001 . The single ANN model was trained as MLP using the Levenberg-Marquardt and Quasi-Newton algorithm separately.

Table 1. Recognition Accuracy of cases in Percentages

\begin{tabular}{|l|l|l|l|}
\hline $\begin{array}{l}\text { Hidden } \\
\text { Nodes }\end{array}$ & $\begin{array}{l}\text { ANN } \\
1\end{array}$ & $\begin{array}{l}\text { ANN } \\
2\end{array}$ & $\begin{array}{l}\text { Combined } \\
\text { ANN }\end{array}$ \\
\hline 10 & 80.7 & 78.2 & $\mathbf{8 1 . 6}$ \\
\hline 11 & 80.4 & 80.0 & $\mathbf{8 5 . 6}$ \\
\hline 12 & 81.3 & 79.6 & $\mathbf{8 5 . 8}$ \\
\hline 13 & 81.3 & 80.7 & $\mathbf{8 8 . 7}$ \\
\hline 14 & 81.6 & 78.4 & $\mathbf{8 5 . 3}$ \\
\hline 15 & 76.7 & 81.6 & 68.2 \\
\hline 16 & 72.2 & 82.0 & 56.7 \\
\hline 17 & 75.8 & 81.8 & 53.6 \\
\hline 18 & 80.7 & 78.4 & 69.6 \\
\hline 19 & 80.0 & 77.3 & 73.6 \\
\hline 20 & 80.2 & 80.9 & 61.9 \\
\hline
\end{tabular}

The network is adjusted based on a comparison of the output and the target until the network output marches the target and the outputs were combined using the averaging method. The dataset were randomly allocated into training $(80 \%)$, validation $(10 \%)$ and testing (10\%). Monitoring and diagnosis performance of the combined pattern recognizer is based on the recognition accuracy and mean square error of the individual and combined accuracies

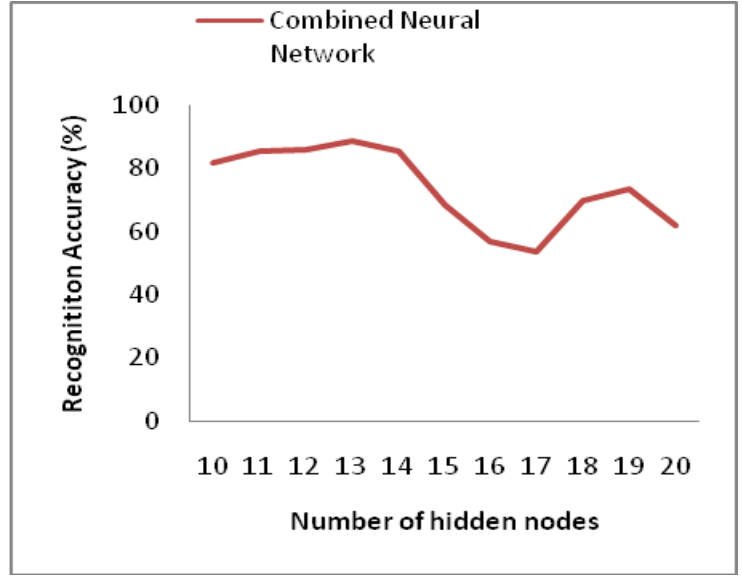

Fig. 1. Recognition Accuracy of combined ANN

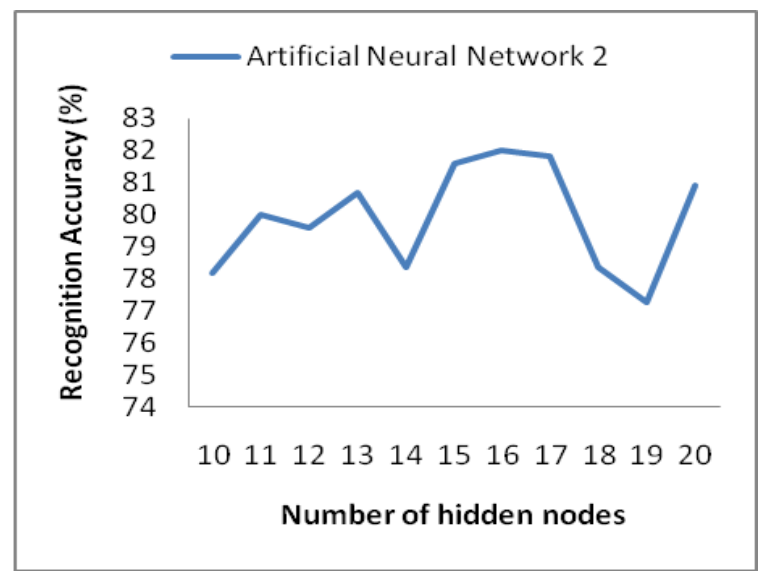

Fig. 2. Recognition Accuracy of ANN trained with Levenberg-Marquardt Algorithm 


\section{RESULTS AND DISCUSSION}

The diagnosis performance of the combined ANN is summarized in Table 1and Table 2 using the idea of [11]. Upon examining Table 1 , it was observed that recognition accuracy of ANN 1 trained with Levenberg (trainlm) is high when the number of nodes is small, however, for a moderate number of nodes the performance is low, for ANN 2 trained with scaled conjugate gradient (trainscg), the performance is almost the same for the varying number of nodes in the hidden layer. For the combined ANN, the performance is high for small hidden nodes, say less than 15 . Similarly from Table 2, the combined ANN gives the least mean square error for smaller hidden nodes compared with the individual ANN model for pattern recognition. Improvement in recognition accuracy of combined ANN for hidden nodes less than or equal to 15 indicates that pattern recognition using combined ANN exhibits a better diagnosis capability to accurately identify source of process mean shift especially for smaller number of nodes in the hidden layer.

Table 2. Mean square error performance of individual and combined recognizer

\begin{tabular}{|l|l|l|l|}
\hline $\begin{array}{l}\text { Hidden } \\
\text { Nodes }\end{array}$ & ANN 1 & ANN 2 & $\begin{array}{l}\text { Combined } \\
\text { ANN }\end{array}$ \\
\hline 10 & 0.1652 & 0.1684 & $\mathbf{0 . 1 6 1 4}$ \\
\hline 11 & 0.1648 & 0.1767 & $\mathbf{0 . 1 4 9 3}$ \\
\hline 12 & 0.1757 & 0.1684 & $\mathbf{0 . 1 5 3 5}$ \\
\hline 13 & 0.1692 & 0.2223 & $\mathbf{0 . 1 6 3 4}$ \\
\hline 14 & 0.1753 & 0.5229 & $\mathbf{0 . 1 6 5 8}$ \\
\hline 15 & 0.1767 & 0.2195 & $\mathbf{0 . 1 7 2 5}$ \\
\hline 16 & 0.2493 & 0.1679 & 0.3169 \\
\hline 17 & 0.2036 & 0.1728 & 0.3064 \\
\hline 18 & 0.1657 & 0.1726 & 0.2242 \\
\hline 19 & 0.1699 & 0.1722 & 0.1426 \\
\hline 20 & 0.2056 & 0.1644 & 0.1460 \\
\hline
\end{tabular}

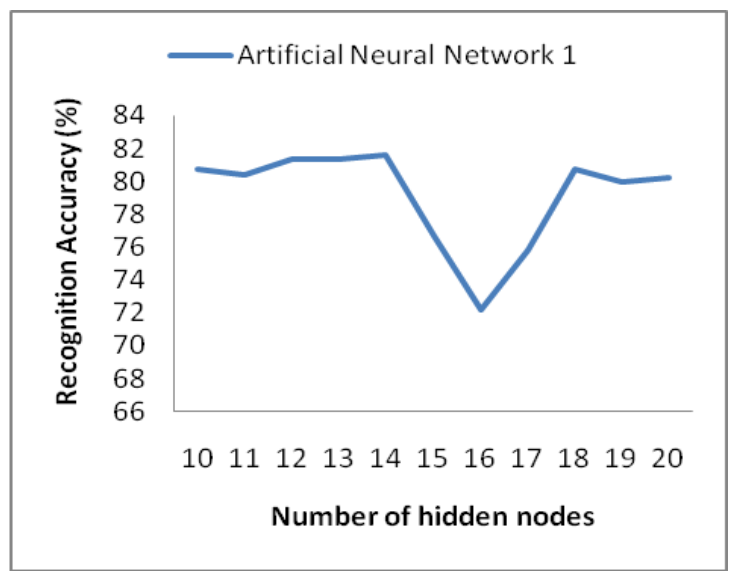

Fig. 3. Recognition Accuracy of ANN trained with QuasiNewton Algorithm

\section{CONCLUSION}

This paper introduces the combined ANN recognizer on varying number of hidden nodes applied to pattern recognition of bivariate process mean shift. It shows that in the design of the combined recognizer, attention need to be drawn to the number of nodes in the hidden layer and reveals that for small number of hidden nodes, the combined ANN model performed better compared to individual model when trained with the same dataset. In the future, this work can be extended to investigate the effect of different dataset allocation in percentages and sample observations on the combined ANN for pattern recognition.

\section{REFERENCES}

[1] Alt, F. B. 1985 "Multivariate Quality Control," Encyclopedia of the Statistical Science (Kotz, S., Johnson, N. L., and Read, C. R. eds.) 6, 110-122

[2] Anagun, A.S. 1998. A neural network applied to pattern Recognition in Statistical Process Contro. Computers and Industrial Engineering 35, 185-188

[3] Chen L.H and Wang T.Y 2004. Artificial Neural Networks to classify mean shifts from multivariate $\chi^{2}$ chart signals. Computers and Industrial Engineering 47 , 195-205.

[4] Crosier R. B. 1988. Multivariate Generalizations of cumulative sum quality control schemes. Technometrics 30, 291-303

[5] Doganaksoy N, Faltin F.W and Tucker W.T 1991, Identification of Out of Control Quality Characteristics in a Multivariate Manufacturing Environment," Communication in Statistics-Theory and Method, 20(9), 2775-2790.

[6] Guh, R.S 2007. Online Identification and Quantification of Mean Shifts in Bivariate Process using a Neural Network-based Approach. Quality and Reliability Engineering International 23, 367-385.

[7] Healy J.D 1987 A note on multivariate CUSUM procedures Technometrics 29,409- 412

[8] Hotelling, H., 1947. Multivariate Quality ControlIllustrated by the Air Testing of Sample Bombsights," Techniques of Statistical Analysis (Eisenhart, C., Hastay, M. W., and Wallis, W. A. eds.), McGraw Hill, New York.

[9] Jackson, J. E. 1985 Multivariate quality control Communications in Statistics- Theory and Methods 14, 2657-2688.

[10] Lowry, C. A., Woodall, W. H., Champ, C. W. and Rigdon, S. E. 1992. A multivariate exponentially weighted moving average control chart. Technometrics $34,46-53$

[11] Maqsood, I., Khan, M.R., and Abraham, A. 2004. An ensemble of neural networks for weather forecasting. Neural Computing and Application 13, 112-122.

[12] Maravelakis P.E, Bersimis S, Panaretos $\mathbf{J}$ and Psarakis S. 2002. Identifying the out of control variable in a multivariate control chart. Communication in StatisticsTheory and Method 31(12), 2391-2408

[13] Mason, R. L., Tracy, N. D. and Young, J. C. 1995. Decomposition of $T 2$ for Multivariate Control Chart Interpretation. Journal of Quality Technology, 27, 99108.

[14] Mason, R. L., Tracy, N. D. and Young, J. C. 1997. A practical approach for interpreting multivariate $T 2$ control chart signals. Journal of Quality Technology, 29, 396-406 
[15] Murphy, B. J. 1987. Selecting out of control variables with the $T 2$ multivariate quality control procedure. The Statistician, 36: 571-583.

[16] Niaki S.T.A and Abbasi B. 2005. Fault diagnosis in multivariate control chart using artificial neural networks. Quality Reliability Engineering International 21,825-840

[17] Pham, D.T and Oztemel, E 1992. Control Chart pattern Recognition using neural networks. Journal of system Engineering. 2, 256-262

[18] Pham, D.T and Wani, M.A.1999. Feature-based control chart pattern recognition. International Journal of Production Research 35(7),1875-1890
[19] Pignatiello, J. J. Jr and Runger, G. C. 1990. Comparisons of multivariate CUSUM charts. Journal of Quality Technology, 22, 173-186

[20] Sepulveda A and Nachlas J.A. 1997. A simulation approach to multivariate control. Computers and Industrial Engineering 33,113-116

[21] Wani ,M.A and Pham, D.T.1999. Efficient control chart pattern recognition through synergestic and distributed artificial neural network. Proc. Instn Mech Engrs 213 Part B, 157-169

[22] Woodall, W.H. and Ncube, M.M. 1985. Multivariate CUSUM quality control procedures. Technometrics, 27 , 285-292. 